\title{
Congenital leukemia presenting as fever in a neonate
}

\author{
Bethany Beard, MD and Joseph Turner, MD \\ Emergency Department, Indiana University School of Medicine, Methodist Hospital, \\ Indianapolis, Indiana
}

\begin{abstract}
Background: Emergency department workup of pediatric fever typically focuses on ruling out serious bacterial infection, but other disease processes can cause fever. Congenital leukemia is a rare but important cause of fever in neonates. We review the presentation, pathophysiology, and potential complications of congenital leukemia presenting to the emergency department as pediatric fever.
\end{abstract}

Case Report: We report a case of a 4-week-old infant brought to the emergency department for fever and "not acting normally." Complete blood count demonstrated hyperleukocytosis. Subsequent bone marrow biopsy and flow cytometry confirmed the diagnosis of congenital leukemia. The extreme elevation of the patient's white blood cell count put her at high risk for complications, necessitating aggressive treatment, close monitoring, and appropriate consultation for comprehensive care.

Why Should an Emergency Physician Be Aware of This: Congenital leukemia is a rare but serious cause of neonatal fever. While the workup for fever without a source in young pediatric patients primarily focuses on ruling out serious bacterial illness, emergency physicians must be familiar with other potentially life-threatening causes of this complaint.

Keywords: leukemia; neonate; fever

This is the author's manuscript of the article published in final edited form as:

Beard, B., \& Turner, J. (2015). Congenital Leukemia Presenting as Fever in a Neonate. The Journal of Emergency Medicine, 48(6), e131-e133. http://doi.org/10.1016/j.jemermed.2015.01.011 


\section{Introduction}

Fever is a common presenting complaint for pediatric patients in the emergency department, accounting for $>10 \%$ of all visits 1, 2 and 3. Emergency department workup of pediatric fever typically focuses on ruling out serious bacterial infections through the use of age-based risk stratification and diagnostic algorithms 4, 5 and 6. However, a number of important noninfectious disease processes can also cause fever in young children 7 and 8.

One rare but important consideration of neonatal fever is malignancy. Congenital leukemia develops while the fetus is in utero and presents within the first 4 weeks of life. The diagnosis of congenital leukemia depends on three major criteria: the presence of immature hematopoietic cells in peripheral blood and bone marrow, hematopoietic cell infiltration into nonhematopoietic

tissue (ie, skin), and exclusion of other conditions in the differential diagnosis (9). This condition has an estimated incidence of $<5$ per 1 million births (10). We report a rare case of congenital leukemia presenting to the emergency department as pediatric fever.

\section{Case Report}

A 4-week-old African-American female presented to the emergency department with parental concerns of fever and "not acting normally.” Her mother noticed decreased activity from her baseline, although she did have good oral intake and urinary output. Earlier in the day, the patient had a measured temperature of $101.5^{\circ} \mathrm{F}$.

The patient was born at $39+5$ weeks. The pregnancy was complicated by untreated maternal group B streptococcus infection and nonactive genital herpes at the time of delivery. The patient was delivered via cesarean section due to persistent decelerations in her heart rate and 
intolerance to labor. After the delivery, the newborn did well and was discharged home under the care of her mother and father.

The patient presented to the emergency department on the day her fever began. On examination, she had a heart rate of 155 beats/min, respiratory rate of 30 breaths/min, temperature of $37.0^{\circ} \mathrm{C}$, and oxygen saturation of $100 \%$. She was alert and well appearing, had normal respiratory effort and clear breath sounds, no cardiac murmur with normal femoral pulses, and no lymphadenopathy or hepatosplenomegaly. Her abdomen was soft and nondistended and she had no skin abnormalities, such as petechiae, bruising, or rash. She also had a strong suck with a pacifier, normal muscle tone, and movement of all four extremities.

The newborn's examination in the emergency department was normal; however, given the findings of fever without a source in this age group, a full septic workup was initiated, including a complete blood count, urinalysis with urine culture, blood culture, and lumbar puncture. The infant was started on empiric ampicillin, cefotaxime, and acyclovir. Laboratory results revealed a white blood cell count of $370,000 \mathrm{k} / \mathrm{mm} 3$ with $86 \%$ blasts, hemoglobin $7.6 \mathrm{~g} / \mathrm{dL}$, and platelet count of $44,000 \mathrm{k} / \mathrm{mm} 3$. Leukemia was strongly suspected; however, further differential diagnosis of hyperleukocytosis includes transient myeloproliferative disease associated with Down syndrome, congenital infections, hemolytic diseases with leukamoid reaction, and other congenital neoplasms, such as neuroblastoma (10). Additional workup was initiated through consultation with pediatric oncology, including uric acid level, lactate dehydrogenase, basic metabolic panel, and ionized calcium to evaluate for tumor lysis syndrome. All additional laboratory results returned normal except an elevated lactate dehydrogenase of 1,795 U/L. The patient did not have tumor lysis syndrome at the time of emergency department presentation and she was ultimately transferred to the associated children's hospital. 
On the morning after hospital admission, the newborn underwent bone marrow biopsy.

Cytogenetic results revealed 46XX, t(4; 11) (q21; 23). She was diagnosed with infant B-cell

acute lymphocytic leukemia, which was later confirmed by flow cytometry. She remained under the care of a pediatric oncologist and received 1 week of prednisone for induction therapy via protocol AALL-0631 with vincristine, daunorubicin, cytarabine, asparaginase, hydrocortisone intrathecal, methotrexate intrathecal, and dexamethasone.

\section{Discussion}

The clinical presentation of congenital leukemia is quite rare and variable. Hepatosplenomegaly is one of the most frequently encountered signs on physical examination, being noted in $80 \%$ of cases (10). Splenomegaly is present in $74 \%$ of cases (11). Lymphadenopathy is observed only one-quarter of the time (10). Patients can also present with other signs and symptoms commonly found in diseases of the bone marrow, including pallor, petechiae, ecchymosis, and abnormal bleeding. Failure to thrive, pleural effusions, and neurologic manifestations have been reported, but are much less common 10 and 11.

A classic manifestation of congenital leukemia is infiltration of leukemic cells into the skin referred to as leukemia cutis. These lesions appear as diffuse palpable violaceous nodules and, when observed, should alert the medical provider to underlying malignancy (9). Leukemia cutis is more frequently observed in acute myelogenous leukemia and is rare in acute lymphoblastic leukemia (9). Reports on the frequency of this finding in congenital leukemia range from 30\% to $60 \% 9$ and 10.

Approximately 70\% of neonatal leukemias are associated with cytogenetic disorders, most commonly with rearrangement of the mixed lineage leukemia (MLL) gene (11q23). 
Translocation of this gene gives rise to fusion proteins that increase leukemogenesis and hematopoiesis (9). The neonate in this case had $46 \mathrm{XX}(4 ; 11)(\mathrm{q} 21 ; 23)$ translocation, which is associated with an aggressive MLL. This particular gene rearrangement is observed in $>60 \%$ of infants with acute lymphocytic leukemia (ALL) and portends a very poor prognosis 12 and 13 . The presence of an MLL gene rearrangement and age $<6$ months are the most important factors predicting poor prognosis, followed by a white blood cell count $>300,000 \mathrm{k} / \mathrm{mm} 3$ and poor response to prednisone (9). Neonates diagnosed with congenital leukemia frequently become refractory to treatment and often die within the first year of life (9).

Leukemia can present with significant leukocytosis and predispose the patient to two severe conditions: thrombosis and tumor lysis syndrome. Initial treatment goals are to avoid such complications by limiting blood viscocity and hyperuricemia from cell destruction. Further emergency department care includes correction of electrolyte abnormalities, including hyperkalemia, ensuring adequate hydration, and treatment of hyperuricemia and coagulation imbalances (14). The patient in this case was at risk for both emergencies, given the severity of her leukocytosis (>300,000 k/mm3).

Hyperleukocytosis is seen in $10 \%$ to $30 \%$ of patients with newly diagnosed ALL (15). High leukemic cell burden increases blood viscosity and causes white blood cell plugging, referred to as leukostasis. The combination of these clinical states increases the risk of strokes. Blast cells also contain pro-coagulant substances that further propagate clot formation and risk of infarction (14). Although leukemic patients may present with anemia, it is important to avoid blood transfusion if possible, as this will further increase blood viscosity. Hyperleukocytosis is primarily treated with induction chemotherapy to decrease the white blood cell count and target leukemic cells in the bone marrow. The neonate in this case began treatment with intravenous 
fluids at twice her maintenance rate to decrease blood viscocity, prevent renal damage, and dilute excess electrolytes.

Leukemic patients undergoing cytoreduction with chemotherapy are at risk of tumor lysis syndrome. This condition occurs due to the destruction of blasts spontaneously or after treatment. Destroyed cells release potassium, phosphate, and uric acid into the serum. Elevated uric acid levels can damage renal tubules and lead to subsequent organ failure. The neonate in this case began early treatment with allopurinol to limit uric acid levels. The addition of sodium bicarbonate to fluids can also help to alkalinize the urine and decrease uric acid crystal deposition (14). Patients with high blood cell destruction might also experience hyperkalemia; therefore, it is important to exclude potassium from intravenous fluid regimens. An electrocardiogram should be obtained early, as hyperkalemia can lead to fatal dysrhythmias. Hyperkalemia in leukemia is treated with standard therapies, including calcium gluconate, insulin, glucose, and beta-agonists.

In cases of newly diagnosed leukemia where tumor lysis or hyperviscocity syndrome might present, it is important to involve a multidisciplinary care team including pediatric oncology, nephrology, and intensive care for further support and treatment recommendations.

\section{Why Should an Emergency Physician Be Aware of This?}

This case is an example of a common emergency department complaint resulting from a rare disease process. The workup and diagnostic approach to neonates with fever is well standardized and directed toward ruling out serious bacterial infections. This case demonstrates the importance of considering not only infectious etiologies, but also hematologic and oncologic 
diseases as the cause of fever. Such hematologic disorders can result in life-threatening conditions that the emergency physician must recognize and treat, including hyperleukocytosis and tumor lysis syndrome, electrolyte imbalances, and organ damage with subsequent failure. 


\section{References}

1. Krauss BS, Harakal T, Fleisher GR. The spectrum and frequency of illness presenting to a pediatric emergency department. Pediatr Emerg Care 1991;7:67-71.

2. Nelson DS, Walsh K, Fleisher GR. Spectrum and frequency of pediatric illness presenting to a general community hospital emergency department. Pediatrics 1992;90:5-10.

3. Massin MM, Montesanti J, Gerard P, Lepage P. Spectrum and frequency of illness presenting to a pediatric emergency department. Acta Clin Belg 2006;61:161-5.

4. Baraff LJ, Bass JW, Fleisher GR, et al. Practice guideline for the management of infants and children 0 to 36 months of age with fever without source. Agency for Health Care Policy and Research. Ann Emerg Med 1993;22:1198-210.

5. Baraff LJ. Management of fever without source in infants and children. Ann Emerg Med 2000;36:602-14.

6. Simon AE, Lukacs SL, Mendola P. Emergency department laboratory evaluations of fever without source in children aged 3 to 36 months. Pediatrics 2011;128:e1368-75.

7. Chow A, Robinson JL. Fever of unknown origin in children: a systematic review. World J Pediatr 2011;7:5-10.

8. Gurion R, Sabella C, Zeft AS. Fever and rash in children: important diagnostic considerations. Minerva Pediatr 2013;65:575-85.

9. Akcakus M, Patiroglu T, Deniz K, Cetin N, Ozdemir MA, Kala M. Congenital acute lymphoblastic leukemia: report of a case with leukemia cutis. Clin Pediatr (Phila) 2004;43:48790. 
10. Van der Linden MH, Creemers S, Pieters R. Diagnosis and management of neonatal leukaemia. Semin Fetal Neonatal Med 2012;17:192-5.

11. Wu X, Du L,Wang X. Congenital monoblastic leukemia presenting as jaundice, pleural effusion, and ascites: case report and literature review. Fetal Pediatr Pathol 2011;30:27-31.

12. Ross JA, Potter JD, Shu XO, Reaman GH, Lampkin B, Robison LL. Evaluating the relationships among maternal reproductive history, birth characteristics, and infant leukemia: a report from the Children’s Cancer Group. Ann Epidemiol 1997;7:172-9.

13. Meyer C, Schneider B, Jakob S, et al. The MLL recombinome of acute leukemia. Leukemia 2006;20:777-84.

14. Werner AH, Scarfone R, Mostoufi-Moab S. A febrile young infant with splenomegaly and ecchymoses. Pediatr Emerg Care 2010;26:442-4.

15. Porcu P, Cripe LD, Ng EW, et al. Hyperleukocytosis leukemias and leukostasis: a review of pathophysiology, clinical presentation and management. Leuk Lymphoma 2000;39:1-18. 